\title{
LES STRATÉGIES INTONATIVES À L'ÉCHANGE ORAL EN SLOVÈNE ET EN FRANÇAIS
}

La linguistique contemporaine de la parole s'intéresse à l'intonation en tant que moyen participant à la réalisation et à la perception des intentions discursives à l'échange oral. Comme les phrases énoncées forment l'ensemble du texte, l'intonation est aujourd'hui perçue comme un moyen autonome contribuant à la construction cohérente des actes de parole spontanés. L'objet de cet article est d'analyser la fonction de l'intonation de l'oral spontané en slovène en comparaison avec les stratégies intonatives à l'oral spontané en français'. A l'intérieur du discours oral, les unités énonciatives sont reliées entre elles par les connecteurs dont le rôle est indissociable de leur forme intonative. C'est pourquoi nous avons entamé une analyse détaillée des facteurs intonatifs et de certaines formes énonciatives (paragraphes oraux et ligateurs) qui créent la spontanéité des messages parlés.

\section{Méthodologie}

\subsection{Le paragraphe oral}

L'unité d'analyse de la parole spontanée qu'introduit M. A. Morel (Morel, DanonBoileau: 1998) par son approche linguistique globale associant prosodie, morphosyntaxe, énonciation et analyse du discours, est appelée paragraphe oral. D'après cette approche, c'est l'unité maximale où l'on peut parler d'une structuration canonique.

Il est défini par l'intonation: la chute conjointe et rapide de l'intensité et de F0 à un niveau bas représente l'indice le plus fiable de la fin du paragraphe ${ }^{2}$. Chaque paragraphe se compose d'un ou de plusieurs constituants. Un paragraphe comprend au moins un rhème précédé, en français, d'un ou de plusieurs préambules, qui sont des segments à valeur thématique et modale. De plus, le paragraphe peut, dans certaines conditions, être de structure ternaire: le rhème est alors suivi d'un postrhème (exemple (1)).

Paragraphe oral type $=$ préambule + rhème + postrhème

1 Cette étude est basée sur les principes théoriques du groupe des chercheurs à l'Université Sorbonne Nouvelle - Paris III qui travaille sous la direction de Mary Annick Morel.

2 Morel, Danon-Boileau (1998), p. 11: «De même qu'à l'écrit le paragraphe ne peut être défini que par des indices typographiques - alinéa au début et blanc à la fin (donc extérieurs au plan segmental) - de même à l'oral seuls les indices suprasegmentaux permettent le découpage en paragraphes». 
(1)

ouais / c'est bon / la tarte Tatin

(Morel, Danon-Boileau, 1998: 155)

Le préambule français est extrêmement décondensé, formé de plusieurs éléments juxtaposés, dont chacun correspond à une fonction énonciative et définie (ligateur, point de vue, modus dissocié, cadre, support lexical disjoint). Il arrive rarement en effet que tous les éléments soient présents dans un seul paragraphe, il peut y en avoir un ou deux seulement.

\subsection{La valeur iconique des indices intonatifs}

M. A. Morel et L. Danon-Boileau partagent le point de vue que l'intonation se voit communément accorder deux fonctions, l'une iconique et l'autre conventionnelle ${ }^{3}$. A partir de ce point de vue, ils proposent l'hypothèse sur la valeur iconique de base de chacun des quatre indices suprasegmentaux qui entrent en jeu dans la mélodie.

La valeur de la hauteur mélodique dérive de la fonction d'appel parce qu'elle permet d'attirer l'attention sur un fragment du discours. La montée mélodique indique ce que l'énonciateur juge négociable, argumentable dans son échange avec l'autre. La chute du fondamental indique à l'inverse un repli sur soi et un désintérêt relatif pour ce que l'autre peut penser de ce qui est dit.

L'intensité, en revanche, indique la façon dont le locuteur gère le canal interactif de l'échange, ainsi que son tour de parole. S'il veut prendre la parole, l'intensité monte, s'il la conserve elle se stabilise, s'il l'abandonne ou s'il considère ce qu'il dit comme un à côté de son discours, elle chute.

La durée relative d'une syllabe indique la représentation que le locuteur se fait de l'état de la formulation des idées que le locuteur s'apprête à exposer sitôt qu'il aura dit ce qu'il est en train de dire. Quand il ne sait comment poursuivre, la durée de la syllabe qu'il profere s'allonge, quand le discours se poursuit simplement, la syllabe conserve la durée des précédentes, et enfin, quand ce qui est dit est l'expression à voix haute d'un monologue intérieur (incise), le débit s'accélère.

La pause silencieuse indique le tournant au sein d'un cadre déja constitué. Sur la base d'une attention accordée, elle met en relief le discours qui va suivre, et permet d'homogénéiser ce qui a précédé ou d'annuler une opération qui a pu être ébauchée.

\subsection{La coénonciation et la co-locution}

A partir des valeurs iconiques de base des quatre indices de l'intonation, M. A. Morel et L. Danon-Boileau proposent la théorie de la coénonciation et de la co-locution. La spécificité de l'échange de l'oral réside, selon eux, dans un double jeu d'an-

3 M. A. Morel et L. Danon Boileau (1998), p. 9: «La fonction conventionnelle est démarcative: elle découpe le continuum de la parole en constituants homogènes. La fonction d'expressivité est, quant à elle, iconique: elle manifeste les émotions du sujet.» 
ticipations: d'une part, celle des attentes que l'énonciateur prête à celui auquel il s'adresse (coénonciation). D'autre part, celle de la révendication du droit à la parole (co-locution).

La gestion de la coénonciation est assurée en large mesure par les variations de F0. Elles explicitent la représentation de la réaction de celui auquel le discours est adressé (considéré comme le coénonciateur). L'énonciateur peut adopter deux positions de la coénonciation:

- soit il prend en compte la position qu'il prête au coénonciateur et, anticipant un désaccord ou une incompréhension, il cherche à obtenir un consensus sur ce qu'il vient de développer: il va se servir alors de la remotée de F0 en plage haute pour l'expliciter;

- soit il ne prend nullement en compte et se moque de ce que celui auquel le discours est adressé peut savoir ou penser ou bien il tient à asserter sa position personnelle. Cela veut dire qu'il se situe en rupture par rapport à la coénonciation, il opère un repli sur soi: il y a alors chute de F0 ou maintien de F0 en plage basse.

La prise en compte du droit à la parole et l'anticipation d'une éventuelle revendication de parole de celui auquel le discours est adressé relèvent la co-locution. Les modulations dans l'attitude colocutive sont marquées par les variations d'intensité.

\section{Analyse du corpus slovène}

\subsection{Circonstances de l'enregistrement}

Dans le cadre de l'étude dont l'objectif est d'analyser l'intonation du slovène spontané par rapport aux caractéristiques du français spontané, deux filles slovènes faisant leurs études à Paris, Ana et Daša, ont été enregistrées le 9 décembre 2001.

L'enregistrement, d'une durée de 25 minutes, a été effectué dans la chambre de l'une des deux interlocutrices à la Cité internationale universitaire de Paris, et à part les filles il n'y avait personne dans la chambre ${ }^{4}$.

\subsection{Caractéristiques et contenu du passage étudiẻ}

De 25 minutes de l'enregistrement, un passage, extrait de la situation «film bosniaque», a été sélectionné pour la présente étude, basée sur 3 minutes de transcription orthographique, dans laquelle 42 secondes ont été analysées sur le logiciel Anaproz.

4 L'embarras du départ se détend tout de suite grâce aux histoires d'Ana qui ne cesse de parler de garçons qu'elle avait rencontrés au cours de la semaine précédente. Comme l'atmosphère est détendue, elles paraissent bien s'amuser pendant leur conversation. Elles se connaissent déjà depuis quelques années et se voient souvent, surtout pour les sorties à Paris. Ana Jakil étudie le piano et la communicologie à Paris XIII et travaille à la télévision autrichienne ORF en tant que journaliste depuis trois ans, ce qui est perceptible aussi sur l'enregistrement. En effet, ses expériences ont sans doute contribué à son comportement détendu devant le microphone. Daša Deželak est étudiante de lettres modernes à Paris III. Elle, par contre, jusqu'à présent, n'a fait aucune expérience dans les médias, et par conséquent, il lui a fallu une dizaine de minutes pour se mettre à l'aise, et encore sa timidité par rapport à Ana reste évidente même à la fin. 
Dans ce passage, il est important de savoir que les locutrices déploient des efforts pour exprimer chacune son point de vue, elles essaient de convaincre l'une l'autre de quel côté il faudrait comprendre ce film sur un soldat bosniaque. En s'adressant constamment l'une à l'autre, elles se servent d'un langage très détendu où l'on trouve beaucoup d'influences de l'anglais ( $k$, sorry etc.), des serbocroatismes (valjda = clair; dans le langage des jeunes), et une syntaxe typique du slovène parlé spontané.

\subsection{Le paragraphe slovène}

En ce qui concerne le paragraphe oral, on va observer comment l'analyse introduite par M. A. Morel est applicable à la langue slovène. Dans les exemples (2a) et (2b), on analyse un segment intonatif en se servant de la méthodologie de M. A. Morel et L. Danon-Boileau ${ }^{5}$ :

a…...h jaz pa ne vem to je meni:.: je to: e me::n jaz: pa te::ga ne verja:\$mem\$\$ a........h je ne sais pas c'est pour moi c'est euh moi:: je ne le crois $\$ p a s \$ \$$ lig. coén. pdv personnel lig.coén.

$\begin{array}{ccc}\text { modus dissocié } & \text { marqueur } & \text { pdv } \\ \text { épistémique } & \text { de rhème } & \text { pers. }\end{array}$

5 Pour pouvoir lire l'exemple (2a) ainsi que les tracés mélodiques de l'exemple (2b), il faut se mettre d'accord sur certaines conventions de transcription:

$\mathbf{S}=$ à l'intérieur d'un énoncé, indique qu'à ce moment une autre voix intervient provoquant un chevauchement de paroles

SS = note la fin de chevauchement de paroles

\{\}$=$ note une pause, même brève; la durée de ces pauses brèves atteignant au maximum une seconde, elles sont notées selon leur importance \{\}$!\{\} /\{\}$

$:=$ note l'allongement d'un son; plus il y a de points plus l'allongement est long $(: /:: /::)$ 
(2b)

ANAPROZ C: $\backslash$ CORPUS F ILM2. WAU 16b $22050 \mathrm{~Hz} 44 \mathrm{~s} .60$

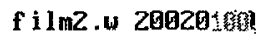
$43 s .99$ brut dyn51DBr le Fel-60 FcFO-300 FQ(350 503 5)

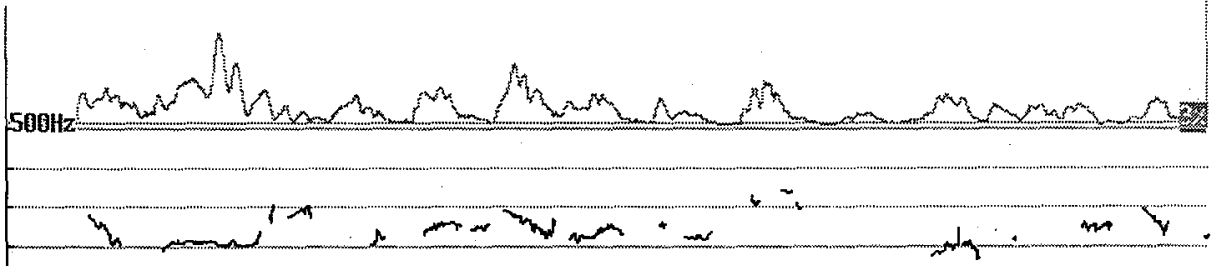

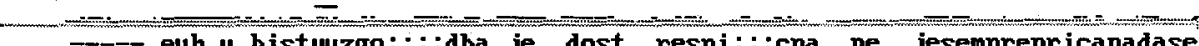
母. $\underbrace{\text { euh u bistuuzgo::: :dba je dost resni:::cna }}_{1 \text {. }}$
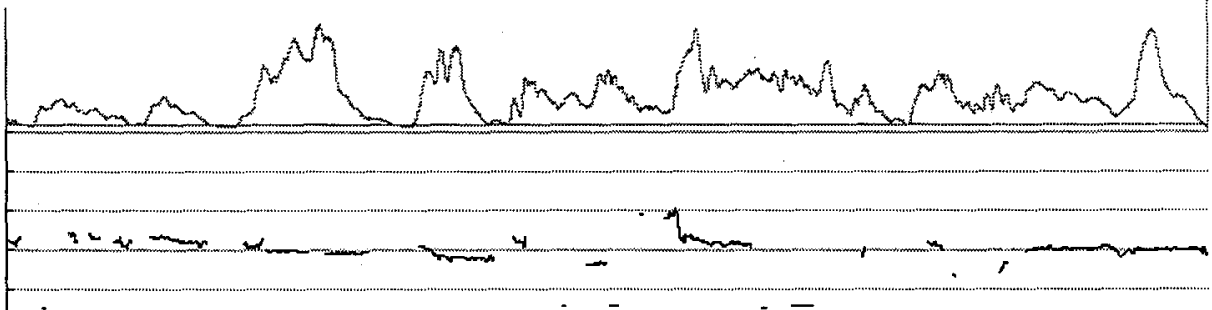

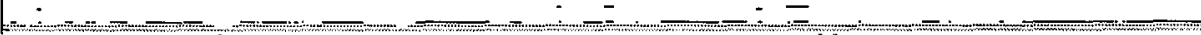
jto: 1 judem:zg\$a::::::::h:: jaz pa ne vem to jemeni::\$\$je to: euh me::n j-az: 3.

AMAPROZ C: \CORPUS $Y$ ILMZ. UAU 16b $2205 B H z$ 44s.90

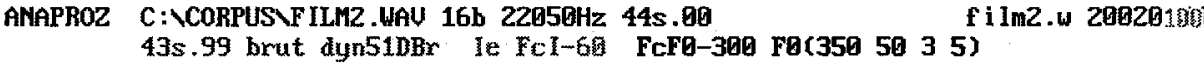

temps $(s.) \longrightarrow$

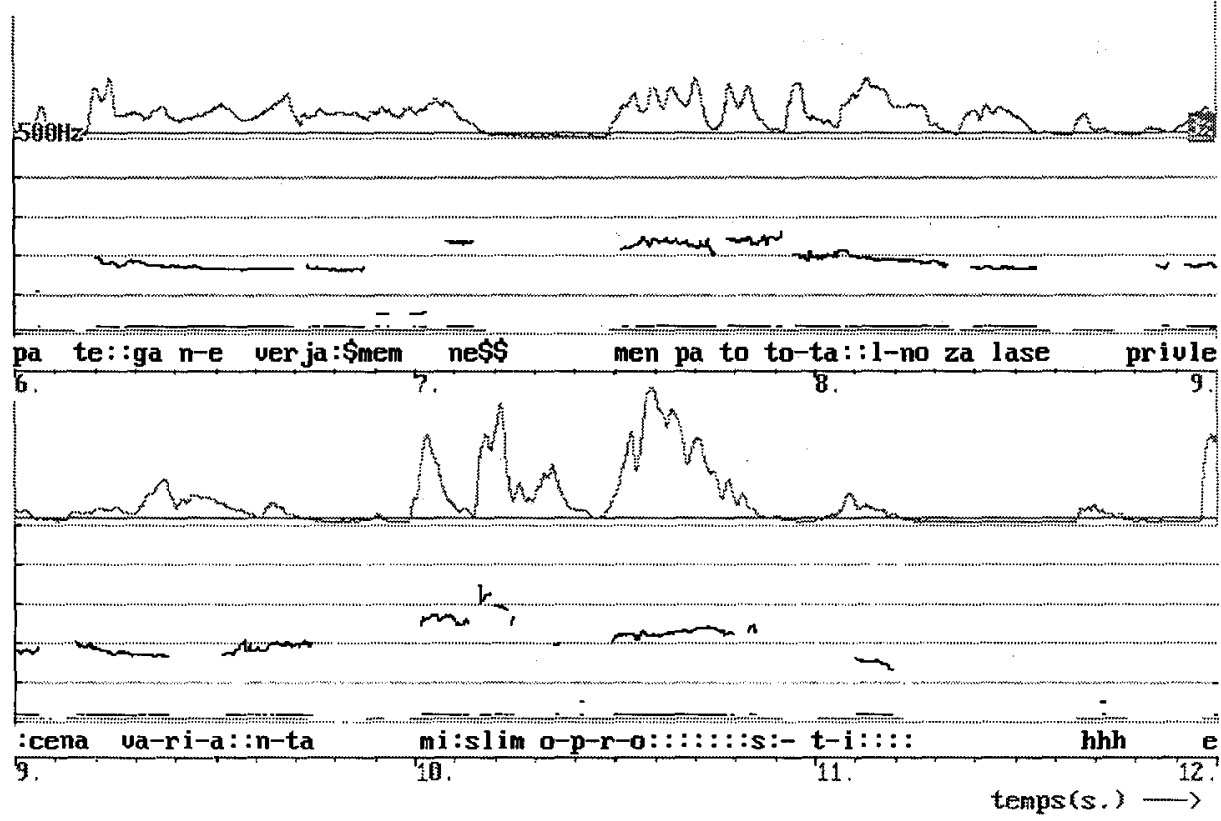


Nous alons analyser maintenant de plus près l'exemple représenté par les schémas $2 a$ et $2 b$ :

Préambule: a:::::::h jaz pa ne vem to je meni:: je to: e me::n (a::....h j'sais pas pour moi c'est ça c'est euh pour moi)

a…:...h $\left(\mathrm{H}_{2}{ }^{6}\right)$ est un ligateur colocutif fortement marqué par une hausse de l'intensité parce qu'à ce moment-là, Ana prend la parole à Daša qui pendant 1,5 seconde essaie encore de garder la parole, ensuite elle se tait.

jaz pa ne vem (j'sais pas moi, H2). Ici, la traduction ne peut pas vraiment correspondre au slovène parce qu'en effet, le pronom personnel n'y est pas deux fois répété, mais au lieu des formes atonne et tonique, juste une particule (pa) met l'accent sur le «moi» qui parle, souvent en s'opposant à quelque chose dit auparavant. L'expression montre le degré de certitude, ou plutôt sa doute sur ce que Daša a dit auparavant. Cela ne veut pas vraiment dire qu'elle ne le sait pas, ce modus dissocié lui sert juste de transition avant d'exprimer son opinion qui est complètement différente de celle de Daša. L'intensité est accentuée sur «jaz» (moi), au niveau des variations de F0, pas de grands changements $(\mathrm{H} 2)$.

to je meni (c'est pour moi) exprime son point de vue personnel, le démonstratif «to» atteint $\mathrm{H} 4$ (consensus), et ensuite, elle lie à cela aussi la proposition suivante, d'ailleurs incomplète:

je to: (c'est) apparaît comme un marqueur de rhème (H3), bien que ce rhème, après, ne soit pas réalisé, parce qu'elle le formule autrement.

e me::n (euh pour moi) de nouveau, elle ne continue pas à base du ligateur et point de vue personnel, mais elle se construit un rhème indépendant.

Rhème: jaz: pa te::ga ne verja:mem $\{30 \mathrm{cs}\}$

(moi je ne le crois pas)

Le rhème est réalisé par une montée d'intensité sur «moi», Ana veut souligner qu'elle n'est pas du tout d'accord avec ce que dit Daša. Le ligateur «pa» a le rôle sémantique proche de «par contre».

Cette analyse montre que le préambule slovène est plus condensé qu'en français, mais il comporte les mêmes éléments: ligateurs coénonciatifs et colocutifs, modus dissocié, point de vue personnel.

En slovène, ainsi qu'en français, l'une des propriétés du préambule représente la rupture dans le fil du discours, c'est-à-dire que le locuteur omet un constituant dont le

6 M. A. Morel et L. Danon-Boileau (1998: 12) identifient le registre intonatif de chaque locuteur et tracent ensuite quatre niveaux sur lesquels ils observent les valeurs des variations de F0:

$\mathrm{H} 4=$ mise en jeu de la coénonciation (convergence/discordance)

$\mathrm{H} 3=$ consensualité acquise

$\mathrm{H} 2=$ repli sur soi

$\mathrm{H} 1$ = rupture de la coénonciation (égocentrage). 
début a déjà été commencé. Cela est exprimé par le marqueur de rhème je to (c'est) qui n'est pas suivi de rhème à cause d'une rupture dans la pensée de la locutrice.

M. Schlamberger Brezar (2000: 69), constate qu'en slovène, le paragraphe oral commence souvent par une particule qui associe les éléments discursifs et modaux (cette particule lie l'énoncé avec ce qui a déjà été dit et exprime la modalité épistémique, exemple (3)):

(3)

A: Meni se to še vedno zdi prisiljevanje ljudi

(moi, je crois toujours que c'est fait pour pousser des gens)

B: Samo, a veš, vseeno je mnogo več ljudi, ki grejo na ekskurzije, kot pa tistih, ki ne grejo. ${ }^{7}$

(mais, tu sais, il y a quand même plus de gens qui vont en excursions que ceux qui ne vont pas)

En ce qui concerne la structure du paragraphe oral, l'analyse a montré que la structure ternaire (préambule + rhème + postrhème), typique pour le français, n'existe pas en slovène.

\subsection{Les ligateurs et les ponctuants en slovène}

La catégorie qu'on a abordée à l'intérieur de l'unité du paragraphe inclut les ligateurs dans le discours spontané. En slovène, il existe des ligateurs qui conviennent parfaitement aux ligateurs français, c'est-à-dire qu'ils ont la même forme intonative et la même position à l'intérieur des constituants du paragraphe. En même temps, on peut les traduire par des expressions qui ont en français le même sens. Dans ce groupe, on peut classifier quelques ligateurs qui régissent la coénonciation, dont M.A. Morel souligne la fréquence de euh en français. En slovène, son équivalent est ainsi le ligateur $e$ parce qu'il marque, lui-aussi, l'hésitation de l'énonciateur marquant à son coénonciateur qu'il a enregistré ce qu'il lui a dit et prépare en même temps ce qu'il a à dire. Elle doit trouver un autre argument pour exprimer son point de vue. La seule différence est liée à la prononciation de ce dernier en slovène: le fait qu'il n'est pas arrondi comme en français s'explique par la caractéristique générale du slovène qui ne connaît pas les voyelles arrondies. Il en va de même pour le ligateur $v$ bistvu - en fait dont la fonction dans la coénonciation s'explique par la mélodie élevée (H3). Le corpus montre, les deux fois que ce ligateur apparaît, que l'intensité reste relativement faible, ce qui veut dire que la locutrice veut atteindre le consensus sur l'objet du discours et non pas prendre ou garder la parole.

Parmi les ligateurs coénonciatifs, on compte aussi quelques modulateurs de la qualification du référent, comme par exemple recimo (disons) ou ne vem (j'sais pas). A cause du rôle qu'ils jouent dans la coénonciation, F0 monte jusqu'à H3.

7 M. Schlamberger Brezar (2000): Skladenjski in pragmatični vidiki povezovalcev v francoskih utemeljevalnih besedilih, thèse de doctorat, Université de Ljubljana, p. 69. 
Le ligateur $a h$, ayant une fonction bien évidente dans la co-locution, ne se caractérise pas uniquement par une forte intensité. En fait, l'intensité se combine avec la durée de $50 \mathrm{cs}$, ce qui facilite à la locutrice la prise de la parole. Même si, une fois le ligateur colocutif prononcé, sa colocutrice essaie de continuer à parler pendant $1,5 \mathrm{sec}-$ onde, la locutrice qui parle avec une intensité plus appuyée va prendre la parole.

Le groupe suivant se compose des ligateurs dont la traduction littérale ne correspond pas à leur fonction dans le discours spontané. C'est le cas du ligateur mislim qui se traduit en français par la 1 ère personne du verbe penser, c'est-à-dire je pense. Pourtant, son emploi courant et ses propriétés intonatives montrent qu'il correspond au ligateur $t u$ vois. Ce ligateur se caractérise dans les deux langues par une mélodie élevée et en même temps par une intensité accrue. Ces caractéristiqes sont liées à son emploi, parce qu'il introduit très souvent une partie sur laquelle le locuteur veut insister davantage et en même temps garder la parole.

Le ligateur razumeš est également très fréquent en slovène, mais celui-ci se traduit soit de la même façon que mislim (tu vois), soit en employant le verbe correspondant à la traduction littérale, tu comprends. Sa forme intonative correspond à celle du ligateur français tu comprends, c'est-à-dire que F0 monte pour que l'énonciateur garde l'attention de celui auquel il s'adresse.

Le ponctuant slovène $n e$, situé à la fin du rhème dans le paragraphe oral, connaît un statut particulier quant à ses correspondants en français. En fait, le corpus étudié montre qu'il peut avoir plusieurs fonctions différentes puisqu'il ne peut pas être dissocié de sa forme intonative:

- s'il est donné en intonation basse, son équivalent sera le ponctuant quoi à la fin du rhème, exprimant un repli sur soi et une attitude égocentré de la part de l'énonciateur,

- une mélodie montante est un indice d'une question,

- si la mélodie monte et qu'il soit en même temps allongé, il correspond au ponctuant hein en français, exprimant que l'énonciateur demande une explication supplémentaire, mais croit encore que le consensus reste possible.

\subsection{La valeur des indices intonatifs en slovène}

En ce qui concerne les indices intonatifs, les premières remarques portent sur les variations de F0. L'analyse du corpus montre que leurs amplitudes ne sont pas si intenses qu'en français, ce qui veut dire que la coénonciation s'opère d'une manière moins évidente qu'en français. C'est aussi la constatation de P. Vitez: «A ce niveau, il $y$ a très peu de différences entre les usages slovène et français des signes intonatifs; elles concernent, généralement, l'intensité des variations des contours tonaux ascendants et descendants. C'est une constatation /.../ qui résulte logiquement du rôle que l'intonation phrastique exerce en français. Il s'agit de la fonction démarcative (délimitation des parties du discours et des segments énonciatifs) que l'intonation phrastique a reprise de l'accent d'intensité. /.../Il s'en ensuit que les réalisations intonatives 
ont en français des variations de F0 plus radicales, les conclusions tonales sont donc relativement plus exprimées qu'en slovène.» 8

La durée de la syllabe dans le discours spontané se distingue également en slovène par rapport aux propriétés temporelles de celle-ci en français. La durée moyenne de la première locutrice est de $24,5 \mathrm{cs}$, et celle de la deuxième de 23,8 . Cela représente une différence en comparaison avec les constatations sur la moyenne de la durée de la syllabe française de $17 \mathrm{cs}$ que M. A. Morel et L. Danon-Boileau avaient faites sur de nombreux corpus français. La situation conversationnelle est détendue, le discours nullement préparé en avance. Pourtant, on pourrait lier les propriétés de la recherche de la formulation en français provoquant la répétition des cumulations des ligateurs dans le préambule avec l'allongement des syllabes en slovène.

La recherche de la formulation joue de même un rôle important quant aux pauses ${ }^{9}$ et aux silences ${ }^{10}$. Dans le corpus slovène, les pauses sont relativement courtes: en moyenne, elles durent $30 \mathrm{cs}$, à l'exception d'une seule de $70 \mathrm{cs}$ (exemple (4)).

mi:slim opro:.......: sti $\{70 \mathrm{cs}\}$ e::.......: $\{40 \mathrm{cs}\}$ če tip pride razumeš pa zgle::da tak kot zgle::.:da pa ma takšen glas $\{30 \mathrm{cs}\}$ pa recimo pride do enega človeka pa mu on//mislim

tu vois excuse-moi $\{70 \mathrm{cs}\}$ euh $\{40 \mathrm{cs}\}$ si le type vient tu comprends et qu'il ressemble à ça et qu'il a une voix comme ça $\{30 \mathrm{cs}\}$ et disons qu'il vient chez quelqu'un $\{25 \mathrm{cs}\}$ et il luil/ tu vois

La fonction de la pause abordée dans l'exemple ci-dessus convient incontestablement à la fonction de la pause-silence en français puisqu'elle permet d'homogénéiser ce qui vient d'être dit et de mettre l'accent sur ce qui va suivre.

\section{Conclusion}

L'étude a montré que l'analyse en paragraphes oraux est applicable aussi à la langue slovène, ce qui met en évidence les points communs du français et du slovène spontanés. Le paragraphe oral en slovène est, comme en français, défini par la chute simultanée de l'intensité et de F0. La divergence se montre dans le préambule qui se caractérise par l'accumulation de ses constituants, mais reste cependant plus condensé qu'en français. De même, le paragraphe oral en slovène ne connaît pas la construction ternaire (préambule, rhème, postrhème), typique pour le français.

8 P. Vitez (1995): Protistavna analiza francoske in slovenske stavène intonacije, doctorat de troisième cycle, Université de Ljubljana, p. 74.

9 M. A. Morel, L. Danon Boileau (1998), p. 41: «La pause courte (20 cs), pause-respiration, brève et biologiquement contrainte, n'a pas de valeur iconique définie».

10 M. A. Morel, L. Danon Boileau (1998), p. 41: «Une pause-silence un peu plus longue (40-80 cs) a une valeur discursive conventionnelle. Elle permet d'unifier ce qui la précède en une sorte de continuum thématique et de rhématiser ce qui va suivre.» 
A l'intérieur du paragraphe oral, nous avons observé de plus près les ligateurs et les ponctuants en slovène pour comparer ensuite leurs propriétés intonatives à celles qui leurs appartiennent en français. Les ligateurs dont le rôle important est évident dans la coénonciation et dans la co-locution forment en général deux groupes: d'un côté, ceux qui correspondent parfaitement aux ligateurs français, et de l'autre côté, ceux dont les propriétés intonatives et par conséquent, leur rôle dans l'échange oral, nous obligent de les traduire par des expressions différentes. C'est le cas de mislim (littéralement je pense) qui correspond à $t u$ vois en français, et du ponctuant ne dont le sens ne peut pas être dissocié de sa forme intonative et correspond par conséquent à quoi, hein ou bien à une question.

Au niveau des valeurs des indices intonatifs, on peut conclure que l'allongement des syllabes en slovène, lié à la recherche de la formulation dans la situation spontanée, correspond à l'accumulation des ligateurs dans le préambule en français. Les amplitudes des variations de F0 sont moins intenses à cause de la fonction démarcative (délimitation des parties du discours et des segments énonciatifs) que l'intonation phrastique a reprise de l'accent d'intensité. Cependant, F0 et l'intensité jouent le rôle principal quant à la coénonciation et à la co-locution lors de l'échange spontané oral.

\section{Bibliographie}

BLANCHE-BENVENISTE, Claire (1991): Le français parlé. Études grammaticales, coll. Sciences du langage, Éditions du CNRS, Paris.

BOLINGER, D. et al. (1972): Intonation. Selected readings, Penguin Books, Baltimore.

BOUVET, D., MOREL, M. A. (2002): Le ballet et la musique de la parole, Ophrys, Paris.

MOREL, M. A., DANON-BOILEAU, L. (1998): Grammaire de l'intonation. Ophrys, Paris.

SCHLAMBERGER BREZAR, M. (2000): Les connecteurs en combinaison avec les marqueurs modaux: l'exemple du français et du slovène. in: Linguistica XL/2, Ljubljana, pp. 273-282.

SCHLAMBERGER BREZAR, Mojca (2000): Skladenjski in pragmatični vidiki povezovalcev v francoskih utemeljevalnih besedilih, doktorska disertacija. Filozofska fakulteta, Univerza v Ljubljani.

VITEZ, P. (1995): Analyse contrastive de l'intonation phrastique en français et en slovène, in: Linguistica XXXV/2, Ljubljana, pp. 257-274.

ZWITTER, A. (2002): Poskus analize stavčne intonacije kot prvine govorne strategije v besedilu, travail de diplôme, Filozofska fakulteta, Univerza v Ljubljani.

\section{Povzetek}

\section{INTONACIJSKE STRATEGIJE GOVORNE IZMENJAVE V SLOVENŠČINI IN FRANCOŠČINI}

Sodobno jezikoslovje govora stavčno intonacijo obravnava kot glasovno sredstvo, ki v tvorbi in zaznavi govornih sporočil sodeluje pri uresničevanju stavčne forme in sporočanjskih namenov. Ker upovedani stavki tvorijo celoto besedila, je tudi intonacija avtonomno jezikovno sredstvo, ki prispeva h gradnji koherentnih spontanih govornih tvorb.

Pričujoča kontrastivna raziskava vloge intonacije $v$ tvorjenju slovenskih in francoskih spontanih govornih tvorb izhaja iz metodologije in izsledkov raziskovalne skupine s Sorbonne Nouvelle Paris III, ki deluje pod vodstvom Mary-Annick Morel. Analiza slovenskega in francoskega govorjenega korpusa vpeljuje razčlembo intonacijskih obrisov na govorjene odstavke in nato še na oblikoskladenjski ravni primerja zgradbo slovenskega in francoskega govorjenega odstavka. Znotraj omenjenih diskurzivnih enot so izpostavljena vezala, ki pri uresničevanju govornih strategij glede na svoje intonacijske značilnosti nastopajo $\mathrm{v}$ dveh vrstah predvidevanj: soizjavljanju in sogovoru. Analiza tako upošteva intonacijske in oblikoskladenjske značilnosti slovenskih in francoskih govornih sporočil ter glede na opisane sporočanjske kriterije predlaga različne prevodne rešitve. 